\title{
A DYNAMIC VARIATION PRINCIPLE FOR ELASTIC-FLUID CONTACTS, APPLIED TO ELASTOHYDRODYNAMIC LUBRICATION THEORY
}

\author{
E. VAN GROESEN and R. VERSTAPPEN \\ Department of Applied Mathematics, University of Twente, P.O. Box 217, 7500 AE Enschede, \\ The Netherlands
}

\begin{abstract}
This paper discusses the variational structure of the line contact problem between an elastic medium and a fluid. The equations for the deformation in the elastic material, and for the flow of the viscous fluid are assumed to be determined from an elastic energy $E$ and a power functional $P$ respectively. Then it is shown that a variational formulation of the combined system can be given: apart from the equations in the interior of both media also the equations expressing balance of forces on the separating boundary are obtained from the power functional
\end{abstract}

$$
P+\frac{\mathrm{d} E}{\mathrm{~d} t}
$$

To that end time dependent deformations are to be considered for which the velocity in the elastic medium vanishes and for which the acceleration of particles on both sides of the common boundary is equal.

This general result is employed in the rest of the paper to a typical problem from elastohydrodynamic lubrication theory. The flow of the lubricant allows a basic variational formulation by assuming it to be dominated by viscous dissipation. The complicated resulting expressions are simplified considerably by imposing the common restriction to small deformations and by exploiting the characteristic length scales of the problem. These approximations are performed directly into the governing power and energy functional. The formulation of the approximated system becomes a genuine variational principle and produces correctly the differential expressions. Moreover, it generates in a natural way efficient numerical methods to calculate the deformation of and the pressure at the free boundary if the time variable is discretized.

\section{INTRODUCTION}

When two bodies in relative motion are pressed together the use of a thin lubricating film can prevent the (dry) contact between the surfaces. The flow of a viscous fluid which forms the separating film causes an elastic deformation of the usually very stiff surfaces. Scaled on the film thickness this effect is of considerable importance in many practical situations. The problem to determine the simultaneous deformation of the surfaces and the flow of the lubricant forms the subject of elastohydrodynamic lubrication (abbreviated EHL) theory.

In this paper the variational structure underlying EHL theory is investigated. A physically realistic variational principle is given without the usual restrictions based on the length scales of the problem. However, if these restrictions are invoked, the approximations can be applied directly in the variational formulation. The resulting approximate functional is related to (but somewhat different from) known variational principles, and the Euler-Lagrange equations are the correct approximate differential expressions.

The search for a variational principle describing the complete system as a whole is motivated by the fact that, separately, both the elastic deformation of the surfaces and the creeping flow of the lubricant can be formulated in terms of an optimization principle. As usual, the elastic deformation should correspond to a state for which the elastic energy $E$ is minimal. In general, a fluid flow problem lacks a variational structure due to the presence of both convection and dissipation. However, the essential and generally adopted assumption (see e.g. [1]) that the effect of convection can be neglected compared to the dissipation allows for a variational description of this so-called creeping flow problem. Unlike the elastic deformation, the equilibrium state in the fluid is not governed by the minimum of an energy, but by the minimum of a power functional $P$.

Here both optimization principles will be combined into one unified formulation. Except from the equations that determine the state and flow in the interior of the media, the main 
feature of the principle is that it also produces correctly the equilibrium of forces on the common boundary.

The general idea of this coupling is first described for a model problem in Section 2. This model problem contains the main constituents of EHL theory but is technically much simpler and more instructive. It turns out that the resulting formulation is not a classical optimization principle for which an extremal value of a certain functional has to be determined. The unified formulation will express that the system is described by that state for which the power functional

$$
P+\frac{\mathrm{d} E}{\mathrm{~d} t}
$$

is minimal.

In Section 3 this concept is worked out for a complete, characteristic EHL problem. Of course, the resulting optimization problem is too difficult to be solved. For this reason the formulation is further approximated on the basis of two assumptions, which are very well accepted at present time. In our opinion it is crucial that these approximations are performed in the variational formulation since this guarantees that the variational structure is maintained. A detailed description of the implications of the two assumptions can be found in Section 4.

In Section 5 the time variable is discretized. This approach generates in a natural way numerical methods. Then the evolution from one time level to a subsequent level is described by a classical optimization problem. From this it is concluded that the steady state equilibrium of the lubricated system is approximately governed by a true, classical variational principle. This provides the time discretized variational formulation of a partial and a posteriori justification. More so, since the resulting, approximate formulation will be interpreted as an integrated form of the balance of mass.

\section{A MODEL PROBLEM}

In this section the basic variational ingredient of the EHL problem is singled out. Omitting various details, the line contact problem between an elastic medium and a fluid can be formulated under certain assumptions as follows.

The deformation of the elastic medium is described in material coordinates $\underline{X}$ by a diffeomorphism $\psi$ mapping the initial configuration $\bar{\Omega}_{0}$ into the current domain $\Omega$. (It is to be remarked here that in the EHL problem $\psi$ is preceded by a rigid rotation.) In the Eulerian picture the force field belonging to the deformation consists of volume forces and of surface forces $\underline{s} \mathrm{~d} l$. Here $\underline{s}(\underline{x} ; n)$ represents the force per unit deformed area at a spatial point $\underline{x}$ on an oriented surface $\mathrm{d} l$ through $\underline{x}$ with positive unit normal $\underline{n}$. The volume forces are neglected here and it is assumed that the steady-state equilibrium of the surface forces in the interior follows from minimizing a potential energy functional. As is common, this functional is assumed to depend on the deformation gradient only:

$$
E(\underline{\psi}):=\int_{\underline{\Omega}_{0}} V\left(\nabla_{\chi} \underline{\psi}\right) \mathrm{d} \underline{X}
$$

With the first variation given by

$$
\begin{aligned}
\delta E(\underline{\psi} ; \delta \underline{\psi}) & =\int_{\bar{\Omega}_{0}} V^{\prime}\left(\nabla_{X} \underline{\psi}\right): \nabla_{X} \delta \underline{\psi} \mathrm{d} X \\
& =\int_{\bar{\Omega}_{0}}-\operatorname{div}_{X} V^{\prime}\left(\nabla_{\bar{X}} \underline{\psi}\right) \cdot \delta \underline{\psi} \mathrm{d} \underline{X}+\int_{\partial \bar{\Omega}_{0}} \delta \underline{\psi} \cdot V^{\prime}\left(\nabla_{X} \underline{\psi}\right) n_{0} \mathrm{~d} l_{0}
\end{aligned}
$$

the equilibrium equation in the interior becomes

$$
-\operatorname{div}_{X} V^{\prime}\left(\nabla_{X} \underline{\psi}\right)=0 \quad \text { in } \bar{\Omega}_{0} .
$$


This Euler equation expresses balance of forces on the boundary of any volume in $\Omega$, i.e.

$$
\int_{\partial A} \underline{s} \mathrm{~d} l=\underline{0} \forall A \subset \Omega,
$$

if the force on a surface in the deformed domain is defined as

$$
\underline{s} \mathrm{~d} l:=V^{\prime}\left(\nabla_{\chi} \psi\right) \underline{n}_{0} \mathrm{~d} l_{0} \circ \underline{\psi}^{-1} \text { in } \Omega .
$$

This leads to the interpretation of the boundary term in (1) as

$$
\int_{\partial \Omega}\left(\delta \underline{\psi}^{\circ} \underline{\psi}^{-1}\right) \underline{s} \mathrm{~d} l .
$$

The fluid under consideration is described directly in Eulerian variables. With $\Sigma$ denoting the fluid domain and $\underline{v(x)}$ the (steady state) velocity field, the assumption of incompressibility requires $\underline{v}$ to satisfy:

$$
\operatorname{div}_{x} \underline{v}=0 \text { in } \Sigma \text {. }
$$

Furthermore it is assumed that the motion is primarily determined by viscous forces. Then, neglecting acceleration (and any other volume) forces, the motion of the fluid is obtained in a Stokes approximation from a power functional. This power is assumed to depend on the (Eulerian) velocity gradients only:

$$
P(p, \underline{v}):=\int_{\Sigma}\left(W\left(\nabla_{x} \underline{v}\right)-p \operatorname{div}_{x} \underline{v}\right) \mathrm{d} \underline{x} .
$$

The Lagrange multiplier $p$ is introduced to take account for the incompressibility condition and will become the pressure later on. The first variation of $P$ with respect to the velocity $\underline{v}$ is given by

$$
\begin{aligned}
& \int_{\Sigma}\left(W^{\prime}\left(\nabla_{x} \underline{v}\right): \nabla_{x} \delta \underline{v}-p \operatorname{div}_{x} \delta \underline{v}\right) \mathrm{d} \underline{x} \\
& \quad=\int_{\Sigma}\left(-\operatorname{div}_{\underline{x}} W^{\prime}\left(\nabla_{\underline{x}} \underline{v}\right)+\nabla_{x} p\right) \cdot \delta \underline{v} \mathrm{~d} \underline{x}+\int_{\partial \Sigma} \delta \underline{v} \cdot\left(W^{\prime}\left(\nabla_{x} \underline{v}\right)-p I\right) \underline{n} \mathrm{~d} l .
\end{aligned}
$$

The vanishing of the first term in the right-hand side of this expression leads to the equation

$$
-\operatorname{div}_{x} W^{\prime}\left(\nabla_{x} \underline{v}\right)=-\nabla_{x} p \text { in } \Sigma .
$$

Consequently, in equilibrium, the first variation reads

$$
\delta P(p, \underline{v} ; \delta \underline{v})=\int_{\partial \Sigma} \delta \underline{v} \cdot\left(W^{\prime}\left(\nabla_{x} \underline{v}\right)-p I\right) \underline{n} \mathrm{~d} l
$$

and $\left(W^{\prime}\left(\nabla_{x} \underline{v}\right)-p I\right) n \mathrm{~d} l$ is to be interpreted as the force on a surface $\mathrm{d} l$ in the fluid. So far for the description of the two media separately.

To investigate the line contact, let $\Gamma$ be the common boundary of the two media, i.e. $\Gamma=\partial \Omega \cap \partial \Sigma$. The no-slip condition for the viscous fluid requires the velocity to be equal to the velocity of the elastic particles on $\Gamma$, that is

$$
\underline{v}=\partial_{t} \underline{\psi} \circ \underline{\psi}^{-1}=\underline{0} \text { on } \Gamma \text {. }
$$

The balance of forces on this free boundary is satisfied if

$$
\left(V^{\prime}\left(\nabla_{\chi} \underline{\psi}\right) n_{0}\right) \circ \underline{\psi}^{-1}=-\left(W^{\prime}\left(\nabla_{x} \underline{v}\right)-p I\right) \underline{n} \text { on } \Gamma \text {. }
$$

It is to be noted that the minus sign in this expression is caused by the fact that the normals $\psi\left(\underline{n}_{0}\right)$ and $\underline{n}$ are opposite: $\underline{n}_{0}$ is the unit outward normal of $\Omega_{0}$ and $\underline{n}$ the unit outward normal of $\Sigma$.

Since each force in the equality (5) appeared in the boundary term of the first variation, this equation is satisfied if the contributions (2) and (3) cancel on $\Gamma$. This leads to the following observation. 
OBSERVATION 2.1. The equations in the interior of the elastic medium and in the fluid, as well as the correct balance of force on the common boundary $\Gamma$ are obtained from

$$
\delta P(p, \underline{v} ; \delta p, \delta \underline{v})+\delta E(\underline{\psi} ; \delta \underline{\psi})=0
$$

for arbitrary functions $\delta \underline{\psi}$ in $\bar{\Omega}_{0}$ and $\delta p$ and $\delta \underline{v}$ in $\Sigma$ subject to the constraint

$$
\delta \underline{v}=\delta \underline{\psi}^{\circ} \underline{\psi}^{-1} \text { on } \Gamma \text {. }
$$

REMARK. It is to be observed that (6) is not a classical variational principle for the complete system: the correct equations (including the free boundary conditions) can not be obtained by a variational procedure for a quantity defined on the union of the two domains. In fact, $\underline{v}$ and $\underline{\psi}$ have to satisfy the relation (4), but the quantities $\underline{\psi}+\delta \underline{\psi}$ and $\underline{v}+\delta \underline{v}$ will not satisfy (4) under the requirement (7).

At first glance, expression (6) does not inherit the physical interpretations of the two constituents $\delta E=0$ (principle of stationary energy) and $\delta P=0$ (stationary power). Physically, the dimensions of the two quantities in equation (6) differ and the same is true for the constraint (7). This discrepancy feeds the conception that a variation of the deformation in $\bar{\Omega}_{0}$ should be viewed as a dynamic change. For this reason a time-dependent deformation $\bar{\Psi}(\underline{X}, t)$ is introduced as

$$
\bar{\Psi}(X, t)=\psi(\underline{X})+t \delta \psi(X)+o(t) .
$$

In terms of the deformation $\underline{\Psi}$, the constraint (7) expresses the fact that the dependence on time is such that the velocity of the motion $\underset{\Psi}{\Psi}(\underline{X}, t)$ equals the variation of the velocity of a fluid particle on the boundary $\Gamma$ :

$$
\delta \underline{v}=\left.\partial_{t} \bar{\Psi} \circ \underline{\Psi}^{-1}\right|_{t=0} \quad \text { on } \Gamma .
$$

Furthermore, the variation of the elastic energy $E$ equals, by definition, the time-rate change of $E(\underline{\Psi}(t))$ at $t=0$. In this way, expression (6) can be given the following formulation.

Proposition 2.1. A map $\bar{\Psi}(t)$ in $\bar{\Omega}_{0}$, a velocity field $\underline{v}$ and a scalar function $p$ in $\Sigma$, related by the no-slip condition (4), satisfy the correct equations in the interior of $\Omega$, in a fluid domain $\Sigma$ and on the common boundary $\Gamma$ if

$$
\delta P(p, \underline{v} ; \delta p, \delta \underline{v})+\left.\frac{\mathrm{d}}{\mathrm{d} t} E(\underline{\Psi}(t))\right|_{t=0}=0
$$

for arbitrary variations $\delta \underline{v}$ in $\Sigma$ and all time dependent deformations $\underline{\Psi}(t)$ subject to $\underline{\Psi}(0)=\underline{\psi}$ and the constraint (8).

Remarks.

(1) Physically, the constraint (8) can be interpreted in an acceptable way as an instantaneous variation of the flow of the lubricant that is attended with a dynamic variation of the deformation of the surfaces.

(2) It is to be noticed that variations of the fluid domain are not considered in this formulation. In particular, the free boundary $\Gamma$, which is the image under the mapping $\psi$ of a part (say $\Gamma_{0}$ ) of the boundary $\partial \bar{\Omega}_{0}$, is fixed. In this sense a variation of the deformation is restricted. This restriction on $\delta \psi$ will now be pointed out more clearly. To that end a variation of the power functional $P$ will also be interpreted as a change in time. For fixed $\Sigma$ take time-dependent pressure and velocity as

$$
\tilde{p}(\underline{x}, t)=p(\underline{x})+t \delta p(\underline{x}) \text { and } \tilde{\underline{v}}(\underline{x}, t)=\underline{v}(\underline{x})+t \delta \underline{v}(\underline{x}) .
$$

Then the time derivative of $P$ is given by

$$
\frac{\mathrm{d}}{\mathrm{d} t} P(\tilde{p}(t), \underline{\tilde{v}}(t))=\int_{\Sigma} \partial_{t}\left(W\left(\nabla_{x} \underline{\tilde{v}}\right)-\tilde{p} \operatorname{div}_{x} \underline{\tilde{v}}\right) \mathrm{d} \underline{x}+\int_{\partial \Sigma}\left(W-\tilde{p} \operatorname{div}_{x} \underline{\tilde{v}}\right) \underline{\tilde{v}} \cdot \underline{n} \mathrm{~d} l .
$$

By taking the no-slip condition (4) into account this expression reduces at $t=0$ to

$$
\left.\frac{\mathrm{d}}{\mathrm{d} t} P(\bar{p}(t), \underline{v}(t))\right|_{t=0}=\delta P(p, \underline{v} ; \delta p, \delta \underline{v}) .
$$


Here an, at this stage, unessential integral lover the boundary $\partial \Sigma / \Gamma$ is dropped. Considering the physical dimensions, the variation $\delta E$ should be a time-rate change of a power in order to add the variations $\delta P$ and $\delta E$. This is achieved by introducing a deformation $\Psi(X, t)$, such that for small $t$

$$
\Psi(X, t)=\psi(X)+\frac{1}{2} t^{2} \delta \psi(X)
$$

Indeed, the first variation of $E$ then reads

$$
\delta E(\psi ; \delta \psi)=\left.\frac{\mathrm{d}^{2}}{\mathrm{~d} t^{2}} E(\Psi(t))\right|_{t=0} .
$$

In this light, the time-dependence of the fluid domain, resulting from the relation $\Gamma=\Psi\left(\Gamma_{0}\right)$ is approximately quadratic. This justifies the following conclusion.

Proposition 2.2. The equations in the interior of the elastic medium and in the fluid as well as the correct balance of forces on the free boundary $\Gamma$ are obtained from

$$
\left.\frac{\mathrm{d}}{\mathrm{d} t}\left(P(\tilde{p}(t), \underline{\tilde{v}}(t))+\frac{\mathrm{d}}{\mathrm{d} t} E(\Psi(t))\right)\right|_{t=0}=0,
$$

for all functions $\bar{p}(x, t), \underline{v}(x, t)$ in $\Sigma(t)$ and $\Psi(\underline{X}, t)$ in $\bar{\Omega}_{0}$ subject to $\tilde{p}(0)=p, \tilde{v}(0)=\underline{v}$ in $\Sigma$, $\Psi(0)=\underline{\psi}, \Psi_{t}(0)=0$ in $\bar{\Omega}_{0}$, and

$$
\partial_{t} \underline{\tilde{v}}=\left.\partial_{t} \underline{\Psi} \circ \underline{\Psi}^{-1}\right|_{i=0} \quad \text { on } \Gamma .
$$

From a physical point of view, equation (10) characterizes the model problem as follows: the deformation $\Psi(t)$ of $\Omega_{0}$ and the velocity $\bar{v}(t)$ and pressure $\bar{p}(t)$ in $\Sigma(t)$ correspond to a state for which the total power

$$
P+\frac{\mathrm{d} E}{\mathrm{~d} t}
$$

is stationary, where the velocity of the elastic motion $\Psi(t)$ must be zero. Moreover, the constraint (11) combined with the no-slip condition $\bar{v}(0)=\underline{v}=\underline{0}$ on $\Gamma$ implies that on the common boundary $\Gamma$ the contact between a fluid and an elastic particle is maintained, since the (velocity and) the acceleration of both particles are identical.

\section{ELASTOHYDRODYNAMIC LUBRICATION}

The basic concepts sketched in the model problem play an important role in EHL theory. The rest of this paper deals with the lubricated line contact between a deformable, rotating cylinder (at angular velocity $\omega$ ) and a rigid surface. The cylinder is so long that it is in a state of plain strain, i.e. the deformation is independent of the axial coordinate, and has no component in this direction. In the undeformed state, the cross-section of the cylinder is described by

$$
\Omega_{0}=\left\{\underline{\xi}=(\xi, \eta) \in \mathbb{R}^{2} \mid-r<\xi<r,-k_{0}(\xi)<\eta<k_{0}(\xi)=\sqrt{ }\left(r^{2}-\xi^{2}\right)\right\}
$$

and the deformation of the elastic material is written as a composition of a rigid motion $\underline{\xi} \rightarrow \underline{X}$ and an elastic deformation $X \rightarrow \underline{x}=\psi(\underline{X})$, where

$$
\underline{R}(\underline{\xi}, t)=\underline{X}=\mathscr{R}(t) \underline{\xi} \quad \text { with } \mathscr{R}(t)=\left[\begin{array}{cc}
\cos \omega t & \sin \omega t \\
-\sin \omega t & \cos \omega t
\end{array}\right] \text { and } \psi(\underline{X})=\underline{X}+\underline{u}(\underline{X})
$$

The deformed cylinder $\Omega$ is separated from the rigid surface $Y=\left\{x=(x, y) \in \mathbb{R}^{2} \mid y=y_{0}(x)\right\}$ by a thin film of lubricant, which is also assumed to be in a state of plain strain.

In the model problem, the fluid was defined by a power density $W\left(\nabla_{x} y\right)$ and the formulation was based on the balance of the surface forces. Usually (see for instance [2]), a fluid is defined 
by a constitutive equation giving the Eulerian stress tensor $S$ (defined by $S(\underline{x}) n:=\underline{s}(\underline{x} ; \underline{n})$ ) as a function of the symmetric part of the gradient of the Eulerian velocity

$$
S=S\left(\nabla_{\underline{x}} \underline{v}+\nabla_{\underline{x}} \underline{u}^{\mathbf{T}}\right) \text { in } \Sigma,
$$

and the equilibrium state is governed by three axioms: (a) the law of conservation of mass, (b) the balance of force, and (c) the balance of moment. This implies that the model problem has to be adapted to arrive at a unified formulation of EHL based on the same concepts. In particular, the volume forces have to be included in the second law of Newton. In the domain $\Sigma$, the three equilibrium equations for the lubricant (with constant mass density $\rho$ ) are, respectively [3],
(a) $\operatorname{div}_{x} \underline{y}=0$,
(b) $\operatorname{div}_{\underline{x}} S=\rho(\underline{y} \cdot \nabla) \underline{v}$, and
(c) $S=S^{\mathrm{T}}$.

It is well-known that these equations can not be found as the Euler-Lagrange equations of a variational principle [4]. However such a principle can be found if the following essential assumption is adopted.

Assumption 3.1. Let $\left|\nabla_{x} \underline{v}\right| \ll 1$, so that the constitutive equation (12) may be linearized

$$
S\left(\nabla_{\underline{x}} \underline{v}+\nabla_{\underline{x}} \underline{v}^{\mathrm{T}}\right)=S(0)+\mathscr{C}\left(\nabla_{\underline{x}} \underline{v}+\nabla_{\underline{x}} \underline{v}^{\mathrm{T}}\right) \stackrel{(13 c)}{=} S(0)+\mu\left(\nabla_{x} \underline{v}+\nabla_{\underline{x}} \underline{v}^{\mathrm{T}}\right),
$$

where the viscosity $\mu$ is constant [2]. Furthermore, assume that Reynolds number is small, that is

$$
\rho h\left(v_{k}+v_{0}\right) / \mu \ll 1 \text {, }
$$

where $h$ is a characteristic length of $\Sigma$ in $y$-direction, and $v_{k}$ and $v_{0}$ are the velocities in $x$-direction on the surfaces $y=k:=\underline{\psi}{ }^{\circ} \underline{R}\left(k_{0}\right)$ and on $Y$ respectively.

Indeed, under this last assumption, the acceleration force $\rho_{0} \phi_{t t}{ }^{\circ} \phi^{-1}=\rho(\underline{u} \cdot \nabla) \underline{v}$ can be omitted from equation (13b) (see [5] for the details) and analogous to the model problem the power functional $P$ can be defined. With assumption 3.1, the Euler-Lagrange equations resulting from arbitrary variations of the power $P$ with respect to $\underline{v}$ and $p$ are the equilibrium equations (13a) and (13b) for the following choice of the power density

$$
W\left(\nabla_{x} \underline{v}\right)=\frac{1}{2} \mu \nabla_{x} \underline{v}:\left(\nabla_{x} \underline{v}+\nabla_{x} \underline{v}^{\mathrm{T}}\right) \text { and } \quad-p I=S(0) \text { in } \Sigma \text {. }
$$

The vector $-p \underline{n}$ can be interpreted as the force per unit area exerted on a surface with unit normal $\underline{n}$ in rest, i.e. when $\nabla_{x} \underline{v}+\nabla_{x} \underline{v}^{\mathrm{T}}=0$. Concerning a fluid in rest, and restricting $p$ to positive values, the force $-p n$ provides the fluid with the characteristic property that it fills the whole volume in which it is captured. On the other hand, if $p \leq 0$ in a volume $A \subset \Sigma$, then $A$ is not completely filled and $\Sigma$ is not an isotropic, homogeneous continuum in which the equilibrium is governed by equation (13). For this reason, the fluid domain is confined to that part of the gap between $\Omega$ and $Y$ where the pressure $p$ is positive. That is, $\Sigma$ is the closure of the set

$$
\stackrel{0}{\Sigma}^{0}=\left\{\underline{x}=(x, y) \in \mathbb{R}^{2} \mid k(x, t)<y<y_{0}(x) \text { and } p>0\right\} .
$$

For the deformation of the cylinder $\Omega_{0}$ it is assumed that the following requirements are satisfied.

Assumption 3.2. Let $\left|\nabla_{\chi} \underline{\underline{u}}\right| \ll 1$, and approximate the energy density $V$ by

$$
V\left(\nabla_{X} \psi\right):=\bar{V}\left(\nabla_{X} \underline{u}\right)=\bar{V}(0)+\bar{V}^{\prime}(0) \nabla_{X} \underline{u}+\frac{1}{2} \nabla_{X} \underline{u}: C \nabla_{X} \underline{u}
$$

where $\bar{V}(0)=0, \bar{V}^{\prime}(0)=0$ and $C=\bar{V}^{\prime \prime}(0)$. Furthermore, assume that

$$
\rho_{0}(\omega r)^{2} \ll|C|
$$

where $\rho_{0}$ denotes the mass density in $\Omega_{0}$ and $\underline{y}_{\omega}(\underline{X}):=\omega(Y,-X)^{\mathrm{T}}$ is the velocity of the rigid rotation $\boldsymbol{R}$. 
The justification of these assumptions is as follows. The potential energy density $V$ will be a function of the gradient of $\psi$ only, since the rigid motion $R$ is nothing but a change of the material frame and thus should not alter the potential elastic energy. Moreover, the fact that both $\bar{V}(0)=0$ and $\bar{V}^{\prime}(0)=0$ is prompted by the observation that the trivial deformation $X \rightarrow X$ does not alter the potential elastic energy $E$, nor does it induce a surface force.

As in the fluid, the balance of moment requires that the stress tensor $\bar{V}^{\prime}(\nabla \underline{u})=C \nabla \underline{u}$ is symmetric. This implies that the 4-tensor $C$ depends on two (Lamè) constants only. Denoting these constants by $\lambda$ and $v$, the elasticity tensor reads $C_{i j n m}=\lambda \delta_{i j} \delta_{n m}+v\left(\delta_{i n} \delta_{j m}+\delta_{i m} \delta_{j n}\right)$, where $\delta_{i j}=0$ for $i \neq j$ and $\delta_{i i}=1$ (see for instance [3]).

Although the potential elastic energy $E$ is not affected by the rigid motion $R$, the total elastic energy does depend on $R$. Indeed, the total energy $E_{\text {tot }}$ consists of the potential energy $E$ and the kinetic energy $K: E_{\text {tot }}=E+K$. The kinetic energy $K$ is approximated on the basis of assumption 3.2 by

$$
K=\frac{1}{2} \int_{\bar{\Omega}_{0}} \rho_{0}\left[\left(\underline{v}_{\omega} \cdot \nabla_{X}\right) \underline{\psi}\right]^{2} \mathrm{~d} \underline{X}=\frac{1}{2} \rho_{0} \pi r^{2}(\omega r)^{2}-\frac{1}{2} \rho_{0} \omega^{2} \int_{\bar{\Omega}_{0}} \underline{X} \cdot \underline{u} \mathrm{~d} \underline{X}, \quad \text { with } \quad \bar{\Omega}_{0}=R\left(\Omega_{0}\right)
$$

Arbitrary variations of the total energy $E_{\text {tot }}$ with respect to the displacement $\underline{u}$ yield

$$
\operatorname{div}_{X} C \nabla \underline{u}=-\rho_{0} \omega^{2} \underline{X} \text { in } \bar{\Omega}_{0} \text { and } C \nabla_{X} \underline{u} \underline{n}_{0}=\underline{0} \text { on } \partial \bar{\Omega}_{0} .
$$

The source term $-\rho_{0} \omega^{2} \underline{X}$, emanated from the variation of the kinetic energy, can be seen as the centrifugal force caused by the rigid rotation. The contribution of this acceleration force to the elastic energy $E_{\text {tot }}$ can be eliminated by splitting the displacement into a homogeneous and an inhomogeneous part, $\underline{u}=\underline{u}_{h}+\underline{u}_{p}$, where $\underline{u}_{p}$ is a solution of problem (17). By imposing $\underline{u}_{p}(\underline{0})=\underline{0}$, this solution is unique and can be calculated analytically:

$$
\underline{u}_{p}(X)=\frac{\rho_{0} \omega^{2}}{8(\lambda+v)}\left(\frac{3 v+2 \lambda}{\lambda+v} r^{2}-\left(X^{2}+Y^{2}\right)\right) X .
$$

Substitution of this expression for $\underline{u}_{\mathrm{p}}$ into the functional $E_{\mathrm{tot}}$ leads to the elastic energy expressed in terms of $\underline{u}_{h}$ only,

$$
E_{\mathrm{tot}}(\underline{u})=E\left(\underline{u}_{h}\right)=\frac{1}{2} \int_{\bar{\Omega}_{0}} C \nabla_{\underline{X}} \underline{u}_{h}: \nabla_{\underline{X}} \underline{u}_{h} \mathrm{~d} \underline{X}
$$

where an inessential constant

$$
\frac{1}{2} \pi r^{2} \rho_{0}(\omega r)^{2}
$$

has been neglected.

At this point both the equilibrium in the fluid and in the elastic cylinder are described separately by an extremal principle. Then the ideas of the previous section can be applied, with the understanding that now the elastic medium rotates.

Before doing so, appropriate boundary conditions on those parts of the boundary different from the line contact, will be specified. On the rigid surface $Y$ the velocity is prescribed because of the no-slip condition, i.e. $v\left(x, y_{0}\right)=\underline{V}_{0}(x)$. The boundary of $\Sigma$ defined by the isobar $p=0$ consists of two parts; the inlet boundary $x=a(y)$ and the outlet, or cavitation, boundary $x=b(y)$, where $a(y)<b(y)$. (It is assumed that these boundaries can be described by a function of $y$ ). The inlet boundary is traditionally not treated as a free boundary, but is taken to be fixed and the velocity on $x=a$ is prescribed by $\underline{v}(a, y)=\underline{V}_{a}(y)$. By contrast, the outlet boundary $x=b$ is considered as a free boundary and is supposed to be free of surface forces: $S n=\underline{0}$ on $x=b$. This cavitation condition has been proposed before by Coyne and Elrod [6] and is physically arguable if the surface tension of the lubricant can be neglected. The displacement $\underline{u}_{h}$ on the upper boundary $Y=-k_{0}(X)$ of the cylinder is approximately zero. Moreover, the surface force $C \nabla \underline{u} \underline{n}_{0}$ vanishes at that part of the lower boundary $Y=k_{0}(X)$ where the pressure is not strictly positive. On the remainder of this boundary the same 
conditions as in the model problem are imposed: the no-slip condition,

$$
\underline{v}=\partial_{t}(\underline{\psi} \circ \underline{R}) \circ \underline{\phi}^{-1}=\left(\underline{v}_{\omega}+\left(\underline{v}_{\iota} \cdot \nabla_{\underline{X}}\right) \underline{u}\right) \circ \underline{\psi}^{-1} \text { on } \Gamma,
$$

and the balance of surface forces.

The deformed boundary $\Gamma=\{\underline{x} \in \Sigma \mid y=k(x)\}$ is described with respect to the undeformed lower boundary of the cylinder and the displacement on this boundary. In first order, i.e. neglecting terms of the order $|\nabla \underline{u}|^{2}$, the boundary $\Gamma$ is described by the function

$$
k(x)=k_{0}(x)+\underline{u}\left(x, k_{0}\right) \cdot \underline{n}_{0},
$$

where $\tilde{n}_{0}$ is the outward normal of $\Omega_{0}$ with length $r / k_{0}$.

Unlike the model problem, the deformation $\psi$ of the elastic cylinder is not described in terms of the material coordinates. However, since the normal component of the velocity $\underline{v}_{\omega}$ vanishes on the boundary $\partial \Omega$, it holds that

$$
\left.\frac{\mathrm{d}}{\mathrm{d} t} E(\underline{\tilde{u}}(t))\right|_{t=0}=\delta E\left(\underline{u}_{h} ; \delta \underline{u}_{h}\right) \quad \text { with } \quad \underline{u}(\underline{X}, t)=\underline{u}_{h}(\underline{X})+t \delta \underline{u}_{h}(\underline{X})+o(t) .
$$

Hence, similar to proposition 2.1, the following formulation of the complete EHL problem can be given.

Proposition 3.1. Under the assumptions 3.1 and 3.2, a steady state $p(\underline{x}), \underline{v}(\underline{x})$ in $\Sigma$ and $\underline{u}_{h}(\underline{X})$ in $\bar{\Omega}_{0}$ of the EHL problem is described by the requirement

$$
\delta P(p, \underline{v} ; \delta p, \delta \underline{v})+\left.\frac{\mathrm{d}}{\mathrm{d} t} E(\bar{u}(t))\right|_{t=0}=0
$$

for arbitrary variations $\delta p, \delta \underline{v}$ in $\Sigma$ and all deformations $\underline{\underline{u}}(0)$ subject to

$$
\underline{\bar{u}}(0)=\underline{u}_{h} \quad \text { in } \bar{\Omega}_{0} \quad \text { and } \quad \partial_{t} \overline{\bar{u}}(0) \circ \underline{\psi}^{-1}=\delta \underline{v} \quad \text { on } \Gamma
$$

and the boundary conditions

$$
\begin{gathered}
\delta p=p=0 \quad \text { on } \partial \Sigma /(\Gamma \cup Y), \\
\delta \underline{v}=\underline{0}, \quad \underline{y}=\underline{V}_{0} \quad \text { on } \mathrm{Y}, \\
\delta \underline{v}=\underline{0}, \quad \underline{v}=\underline{V}_{a} \quad \text { on } \partial \Sigma \cap\{x=a(y)\} \\
\delta_{t} \bar{u}(0)=\underline{\bar{u}}(0)=\underline{0} \quad \text { on } \partial \bar{\Omega}_{0} \cap\left\{Y=-k_{0}(X)\right\} .
\end{gathered}
$$

Moreover, as in the model problem, the first variation of the power $P$ can be seen as the time derivative at $t=0$ of the functional $P(\bar{p}(t), \bar{v}(t))$. Formally, this derivative is given by

$$
\left.\frac{\mathrm{d}}{\mathrm{d} t} P(\tilde{p}(t), \bar{v}(t))\right|_{t=0}=\delta P(p, \underline{v} ; \delta p, \delta \underline{v})+\int_{\partial \Sigma}\left(W\left(\nabla_{x} \underline{v}\right)-p \operatorname{div}_{x} \underline{v}\right) \underline{v} \cdot \underline{n} \mathrm{~d} l,
$$

and the assertion follows from the remark that the boundary integral can be neglected on basis of assumption 3.1. Further, following the view reflected in proposition 2.2, an alternative description of the EHL problem reads:

Proposition 3.2. Provided that the assumptions 3.1 and 3.2 are fulfilled, the correct equations governing the EHL problem are obtained from

$$
\left.\frac{\mathrm{d}}{\mathrm{d} t}\left(P(\tilde{p}(t), \underline{\tilde{v}}(t))+\frac{\mathrm{d}}{\mathrm{d} t} E(\underline{\bar{u}}(t))\right)\right|_{t=0}=0,
$$

for all functions $\underline{\bar{u}}$ in $\overline{\mathbf{\Omega}}_{0}$ and $\tilde{\bar{p}}, \underline{\tilde{v}}$ in the fluid domain

$$
\Sigma(t)=\left\{(x, y) \in \mathbb{R}^{2} \mid k_{0}+\underline{u}\left(x, k_{0}, t\right) \cdot \underline{\tilde{n}}_{0}<y<y_{0} \quad \text { and } \quad a(y)<x<b(y)\right\}
$$

satisfying

$$
\begin{array}{ccc}
\tilde{p}(0)=p, & \underline{\tilde{v}}(0)=\underline{v}, \quad \underline{\bar{u}}(0)=\underline{u}, \quad \underline{u}_{t}(0)=\underline{0}, \\
\partial_{t} \underline{\tilde{v}}=\left.\partial_{t t} \bar{u} \circ \psi^{-1}\right|_{t=0} & \text { on } \Gamma,
\end{array}
$$


and the following boundary conditions

$$
\begin{array}{rll}
\partial_{t} \tilde{p}(0)=0, & \tilde{p}(0)=0 & \text { on } \partial \Sigma /(\Gamma \cup Y), \\
\partial_{t} \bar{v}(0)=\underline{0}, & \underline{\tilde{v}}(0)=\underline{V}_{0} & \text { on } Y, \\
\partial_{t} \bar{v}(0)=\underline{0}, & \tilde{\underline{v}}(0)=\underline{V}_{a} & \text { on } \partial \Sigma \cap\{x=a(y)\}, \\
\partial_{t t} \underline{\tilde{u}}(0)=\underline{0}, & \underline{\tilde{u}}(0)=0 & \text { on } \partial \bar{\Omega}_{0} \cap\left\{Y=-k_{0}(X)\right\} .
\end{array}
$$

\section{AN APPROXIMATE FORMULATION}

The simultaneous solution of both the flow of the lubricant and the deformation of the cylinder forms an enormous task. In practice, this task is relieved by simplifying the formulation. In broad outline, the extremizing velocity is approximated by function of the pressure in $\Sigma$ and the extremizing displacement by a function of the pressure at $\Gamma$. Then these solutions are substituted into the power functional

$$
P+\frac{\mathrm{d} E}{\mathrm{~d} t}
$$

and there results a functional depending on the pressure only. The variational problem reduces in that way to the problem of finding a stationary point of the power functional with respect to the scalar $p$.

The simplification is based on the assumption that the film thickness is small compared with the length of the lubricated contact [1]:

Assumption 4.1.

$$
\varepsilon:=\frac{\hbar}{l} \ll 1,
$$

where $h$ and $l$ are the characteristic lengths of $\Sigma$ in $y$ - and $x$-direction respectively.

The approximation is performed in the variational formulation. The first step is to simplify the power of the flow.

LEMMA 4.1. The functional $P(p, \underline{v})$ is given, in lowest order of $\varepsilon$, by

$$
P(p, y)=\int_{\Sigma}\left(-p \operatorname{div}_{x} \underline{y}+\frac{1}{2} \mu\left(\partial_{y} v_{1}\right)^{2}\right) \mathrm{d} y \mathrm{~d} x
$$

Proof. Let $v$ represent a characteristic velocity in the horizontal $(x-)$ direction. Then the equation of continuity $(\delta p)$ dictates that the characteristic velocity across the film is of the order $\varepsilon v$. In terms of the scaled, non-dimensional variables $\bar{x}=x / l, \bar{y}=y / h, \bar{v}_{1}=v_{1} / v$, and $\bar{v}_{2}=v_{2} /(\varepsilon v), P$ can be written as (see [7] for details)

$$
\mu v^{2} \int\left[\frac{-h}{\mu v} p\left(\frac{\partial \bar{v}_{1}}{\partial \bar{x}}+\frac{\partial \bar{v}_{2}}{\partial \bar{y}}\right)+\frac{1}{2 \varepsilon}\left(\frac{\partial \bar{v}_{1}}{\partial \bar{y}}\right)^{2}+\varepsilon\left(\left(\frac{\partial \bar{v}_{1}}{\partial \bar{x}}\right)^{2}+\frac{\partial \bar{v}_{1}}{\partial \bar{y}} \frac{\partial \bar{v}_{2}}{\partial \bar{x}}+\varepsilon\left(\frac{\partial \bar{v}_{2}}{\partial \bar{y}}\right)^{2}+\frac{\varepsilon^{2}}{2}\left(\frac{\partial \bar{v}_{2}}{\partial \bar{x}}\right)\right)\right] \mathrm{d} x
$$

Then, the approximation (27) is derived by deleting all terms of the order $\varepsilon$ and higher.

It is convenient to analyze the consequences of this approximation by first neglecting the deformation of the cylinder. Then proposition 3.2 deals with critical points of $P(p, y)$, and the discussion focuses on the flow of the lubricant between the surfaces $y=k(x)=k_{0}(x)$ and $y=y_{0}(x)$. The velocity on these two surfaces is prescribed by the no-slip condition, i.e. the horizontal (=x-direction) components are given by $v_{k}=\omega k_{0}$ and $v_{0}$ respectively. Taking variations of (27) with respect to $v_{2}$ it follows that $\partial_{y} p=0$ in $\Sigma$. Hence isobars are straight lines. In particular, the part of the boundary of $\Sigma$ at which $p=0$ is straight. Consequently, the inlet $(x=a)$ and outlet boundary $(x=b)$ are independent of $y$. Next, variations of (27) with respect to $v_{1}$ lead to

$$
\partial_{x} p=\mu \partial_{y y}^{2} v_{1}
$$


The solution of this equation, supplemented by the no-slip conditions, is given by

$$
v_{1}(x, y)=\frac{1}{2 \mu}\left(y-y_{0}\right)(y-k) \partial_{x} p+\frac{(y-k)}{h} v_{0}+\frac{\left(y_{0}-y\right)}{h} v_{k},
$$

where the film thickness $h$ is introduced according to

$$
h(x)=y_{0}(x)-k(x) .
$$

Furthermore, in the approximation 4.1 , the viscous force on the outlet boundary is dominated by $\mu \partial_{y} v_{1}$. Since this boundary is a straight line, it follows that $v_{k}=v_{0}$ at $x=b$. Exploiting the results so far, part of the integration in (27) can be performed explicitly, i.e.

$$
P(p)=\frac{1}{2} \int_{a}^{b}\left(\frac{h^{3}}{12 \mu}\left(p^{\prime}\right)^{2}-\left(v_{k}+v_{0}\right) h p^{\prime}-\mu \frac{\left(v_{k}-v_{0}\right)^{2}}{h}\right) \mathrm{d} x-\int_{\Gamma} p \underline{y} \cdot n \mathrm{~d} l .
$$

According to the no-slip condition, the last term vanishes:

$$
\underline{v} \cdot \underline{\tilde{n}}=\partial_{t} k=0 \quad \text { on } \Gamma \text {, where } \underline{\tilde{n}}=\left(-\partial_{x} k, l\right)^{\mathrm{T}} \text {. }
$$

This proves:

PROPOSITION 4.1. The approximate solution of the hydrodynamic lubrication problem minimizes $P(p, b)$, where the power functional $P$ is given by $(29)-(30)$, and the variations of the pressure are subject to $p(a)=p(b)=0, p(x)>0$ for $a<x<b$, and $v_{k}=v_{0}$ at $x=b$.

It is to be noticed that a variation with respect to the free boundary $b$ is introduced. This variation replaces the variation of the velocity at the outlet boundary in proposition 3.2 , and leads to the conclusion that the outlet boundary is (approximately) free of forces: $p_{x}(b)=0$. (A more detailed discussion of this variational principle can be found in [8].) If the sliding velocity $v_{k}-v_{0}$ is zero the power functional (29) coincides with previous variational formulations (cf. [9]). Here, (29) is derived from a sound physical principle for the power functional. As will become more clear in the EHL problem, this is to be preferred above the ad hoc way to invent a functional that produces the correct Euler-Lagrange equations, as is common in practice. The additional term in (29) will probably effect the analysis for the optimal shape of bearings (cf. [10]).

Returning to the EHL problem, the assumptions 3.2 and 4.1 simplify the no-slip condition on $\Gamma$ :

LEMMA 4.2. Under assumptions 3.2 and 4.1 the first component of the velocity on the boundary $\Gamma$ is given by

$$
v_{1}=v_{k}\left(=\omega k_{0}\right)
$$

Proof. The no-slip condition (20) reads $\underline{y}=\left(\underline{y}_{\omega}+\left(\underline{v}_{\omega} \cdot \nabla\right) \underline{u}\right) \circ \underline{\psi}^{-1}$ on $\Gamma$. Since $\varepsilon\left|v_{1}\right|=\left|v_{2}\right|$, and $|\nabla \underline{u}| \ll 1$ it holds that

$$
v_{1}(x, k) \simeq(1+O|\nabla \underline{u}|) \omega k_{0} \circ(I-u)(x, k) \simeq \omega k_{0}(x),
$$

which proves the lemma.

Lemma 4.2 gives an approximation of the velocity on the boundary $\Gamma$. This implies that the deformation of $\Gamma_{0}$ can be approximated too.

Corollary.

$$
\frac{\mathrm{d}}{\mathrm{d} X} \underline{u} \cdot \underline{\tilde{n}}_{0}\left(X, k_{0}(X)\right)=\frac{\mathrm{d}}{\mathrm{d} X} u_{2}\left(X, k_{0}(X)\right) .
$$

Proof. If the velocity $y$ on $\Gamma$ is given by the no-slip condition (20), and the deformed boundary is approximated by equation (21), the relation (30) follows from a straight forward substitution. The proof is completed by the observation that (31) is the necessary and sufficient condition equation (30) to hold true if the first component of $\underline{v}$ is approximated as in lemma 4.2. 
This corollary implies that the film thickness can be expressed in terms of the second component of the displacement at $\Gamma_{0}$. Indeed,

$$
h=y_{0}-k_{0}-\left.\underline{u} \cdot \underline{n}_{0}\right|_{r_{0}}=y_{0}-k_{0}-\frac{\beta r^{2}}{k_{0}}-\left.\left(\underline{u}_{h}\right)_{2}\right|_{r_{0}}-c .
$$

The constant $\beta$ depends on the angular velocity and on elastic properties:

$$
\beta=\rho_{0}(\omega r)^{2} /\left(8\left(\lambda_{s}+\mu_{s}\right)\right)
$$

The integration constant $c$ can be interpreted as the change of the distance between the centre of the cylinder and the rigid surface $Y$. Since here this distance is fixed, $c \equiv 0$.

So far for the approximation of the power of the lubricant and the no-slip condition on the common boundary $\Gamma$. With these simplifications, proposition 3.2 leads to an approximate formulation.

In this approximation, the Euler-Lagrange equations resulting from arbitrary variations of the total power

$$
P+\frac{\mathrm{d} E}{\mathrm{~d} t}
$$

with respect to $\delta \underline{v}$ in $\Sigma$ and $\delta \underline{u}_{h}$ in $\Omega_{0}$, are:

$$
\begin{cases}\operatorname{div} C \nabla \underline{u}_{h}=\underline{0} \quad \text { in } \bar{\Omega}_{0} & \partial_{x} p-\mu \partial_{y y}^{2} v_{1}=0 \text { in } \Sigma, \\
\left(C \nabla \underline{u}_{h} \underline{n}_{0}\right) \circ \underline{\psi}^{-1}=+p \underline{n}-\left[\begin{array}{c}
\mu \partial_{y} v_{1} \\
0
\end{array}\right] & \text { on } \Gamma, \text { and } C \nabla \underline{u}_{h} \underline{n}_{0}=\underline{0} \text { on } \partial \bar{\Omega}_{0} / \Gamma_{0} .\end{cases}
$$

With lemma 4.2 it follows that the solution of (33) is again given by (28). With regard to the forces on the boundary $\Gamma$, the following assertion can be proved.

LEMMA 4.3. The $x$-component of the force on the free boundary $\Gamma$ can be neglected compared to the $y$-component. That is

$$
-p n_{1}+\mu \partial_{y} v_{1}=\mathscr{O}(\varepsilon) p n_{2}
$$

Proof. Since $n_{1}=-\partial_{x} k n_{2}$ and $\partial_{x} k=-\partial_{x} h=O(\varepsilon)$ it follows immediately that $p n_{1}=O(\varepsilon) p n_{2}$. Furthermore, in terms of the scaled coordinates introduced in the proof of lemma 4.1, equation (33) reads

$$
\varepsilon \partial_{\bar{x}} p-\partial_{\bar{y}}\left(\mu \partial_{y} v_{1}\right)=0 \text { in } \Sigma .
$$

The operators $\partial_{x}$ and $\partial_{y}$ are scaled and approximately of the same order. This shows that $\mu \partial_{y} v_{1}=O(\varepsilon) p$ in $\Sigma$, and in particular on $\Gamma$.

Remark. At this point it is to be emphasized that the approximation (35) is consistent with (28) if the sliding velocity $v_{k}-v_{0}$ vanishes. This condition has been imposed at the cavitation boundary $x=b$. In addition, assumption 3.1 states that the velocity gradients are small so that the vanishing of the sliding velocity is satisfied on all of the boundary $\Gamma$.

Using the approximation (35), the $y$-component of the solution of the boundary value problem (34) can be written as $\left(\underline{u}_{h}\right)_{2} \circ \psi^{-1}=\mathscr{L}(p)$, where $\mathscr{L}$ is some linear operator. Substituting this into the elastic power

$$
\frac{\mathrm{d} E}{\mathrm{~d} t},
$$

an expression in terms of $\mathscr{L}(p)$ on the boundary $\Gamma$ results:

$$
\begin{aligned}
\frac{\mathrm{d}}{\mathrm{d} t} E(\underline{\tilde{u}}(t)) & =\frac{1}{2} \frac{\mathrm{d}}{\mathrm{d} t}\left[\iint_{\bar{\Omega}_{0}} \operatorname{div} C \nabla \bar{u} \cdot \underline{\tilde{u}} \mathrm{~d} \Omega_{0}+\int_{\partial \bar{\Omega}_{0}} C \nabla \bar{u} \underline{n}_{0} \cdot \bar{u} \mathrm{~d} l_{0}\right] \\
& =\int_{\Gamma} \tilde{p} \partial_{t} \bar{u}_{2} \circ \underline{\psi}^{-1} \mathrm{~d} x=\int_{\Gamma} \tilde{p} \mathscr{L}(\tilde{p}) \mathrm{d} x
\end{aligned}
$$


For this reason, only the value of $\mathscr{L}(p)$ on $\Gamma$ is required. As usual in contact mechanics, this quantity is approximated by solving (34) with the domain $\Omega_{0}$ replaced by a half-plane (see e.g. [11]). The displacement is then approximated by:

$$
\left(u_{h}\right)_{2}^{\circ} \psi^{-1}=\gamma L(p) \text { on } \Gamma
$$

where the constant $\gamma$ is given by $\gamma=(\lambda+2 v) /(2 \pi v(3 \lambda+2 v))$ and the integral operator $L$ is defined as

$$
L(p)(x)=\int_{a}^{b} p(s) \log |x-s| \mathrm{d} s
$$

Finally, the combination of this simplification and the approximated power functional (29)-(30) leads to:

Proposition 4.2. The solution $p(x)=\tilde{p}(x, t=0), b=\tilde{b}(t=0)$ of the EHL problem satisfies (in the approximation under consideration)

$$
\left.\frac{\mathrm{d}}{\mathrm{d} t} \int_{a}^{\tilde{b}}\left[\frac{\tilde{h}^{2}}{12 \mu}\left(\partial_{x} \tilde{p}\right)^{2}-\left(v_{k}+v_{0}\right) \tilde{h} \partial_{x} \tilde{p}-\mu \frac{\left(v_{k}-v_{0}\right)^{2}}{\tilde{h}}\right] \mathrm{d} x\right|_{i=0}=0
$$

with

$$
\tilde{h}(t=0)=y_{0}-k_{0}-\frac{\beta r^{2}}{k_{0}}-\gamma L(p), \quad \text { and } \quad \partial_{t} \tilde{h}(t=0)=0
$$

where the variations are subject to $\tilde{p}(a, 0)=\tilde{p}(b, 0)=0, \tilde{p}(x, 0)>0$ for $a<x<\tilde{b}$ and $v_{k}=v_{0}$ at $x=5$.

Proof. After combining the approximations of $P$ and

$$
\frac{\mathrm{d} E}{\mathrm{~d} t}
$$

the proof of this assertion is completed by the observation that the constraint (25) implies that

$$
\frac{\mathrm{d}}{\mathrm{d} t} \int_{\Gamma}\left(\partial_{t} \bar{u}_{2} \circ \psi-\tilde{v} \cdot \underline{n}\right) \tilde{p} \mathrm{~d} l_{l=0}=0
$$

REMARKS.

(1) It is to be observed that (37)-(38) is a quasi-variational principle: the correct Euler-Lagrange equation is obtained from variations of the pressure $p$ while keeping the quantity $h(p)$ fixed. Stated differently, variations with respect to the free boundary $\Gamma$, via the film thickness, are suppressed.

(2) Another observation is that the formulation (37)-(38) is correct if the assumptions 3.1, 3.2 and 4.1 are satisfied. In the recent liteature many more assumption are stated. (For instance, [12] gives five additional assumptions.) However, all of these are implications of the three earlier mentioned basic assumptions. In particular, the fact that the pressure is independent of the $y$-coordinate needs not to be assumed, but follows from assumptions 3.1 and 4.1 .

Performing the time-differentiation in expression (37) leads to

$$
\left[\frac{h^{3} p^{\prime}}{12 \mu}-\frac{1}{2}\left(\left(v_{k}+v_{0}\right) h\right)\right]^{\prime}=0 \text { for } a<x<b \quad \text { and } \quad p^{\prime}(b)=0 .
$$

This differential equation is known as Reynolds equation (cf. [12]); the free boundary condition is called the Swift-Sommerfeld (or Reynolds) condition. The fact that these physically well-understood and accepted expressions have been derived here, provides a formal, and partial, a posteriori justification of the validity of (37). 


\section{A DISCRETE-TIME APPROACH}

From a numerical point of view, it is natural to interpret the solution $p$ as the limit of a sequence $p^{(0)}, p^{(1)}, p^{(2)}, \ldots$, which is generated iteratively by

$$
p^{(i+1)} \leftarrow \min \int_{a}^{b}\left[\frac{h^{3}}{12 \mu}\left(p^{\prime}\right)^{2}-\left(v_{k}+v_{0}\right) h p^{\prime}-\mu \frac{\left(v_{k}-v_{0}\right)^{2}}{h}\right] \mathrm{d} x,
$$

where $h=h\left(p^{(i)}\right)$, and $p^{(0)}=$ given.

In the light of the previous sections, this iteration can be seen as a process which takes place in time. That is, with $\tau$ an arbitrary, positive time-step, the identification

$$
p^{(0)}=\hat{p}(t=0), \quad p^{(1)}=\hat{p}(t=\tau), \quad p^{(2)}=\hat{p}(t=2 \tau), \ldots,
$$

is made, where $\hat{p}$ denotes the time-discretized pressure. In this way, however one can not satisfy the condition $\partial_{t} h=0$. Indeed, the time-rate change of the film thickness is given by

$$
-\frac{\gamma}{\tau} L(\hat{p}(i \tau)-\hat{p}((i-1) \tau))
$$

which does not vanish in general. For this reason, a different approach is desired. This is possible by an approach that is based on a time-discretization of the variational principle which states that the total power of the lubricated system should be stationary. Then the power functional is discretized like

$$
P(\hat{p}(t+\tau), \hat{v}(t+\tau))+\frac{E\left(\hat{a}_{h}(t+\tau)\right)-E\left(\hat{a}_{h}(t)\right)}{\tau},
$$

where the velocity of the fluid has to be discretized too. Therefore, if $\phi$ is the map that governs the motion of the fluid, the velocity in $\Sigma$ is written as

$$
\hat{y}(\underline{x}, t+\tau)=\frac{\phi(\xi, t+\tau)-\phi(\xi, t)}{\tau} \text { with } \underline{x}=\phi(\underline{\xi}, t+\tau) \text {. }
$$

Recalling observation 2.1 , the time-discretized variational formulation reads: given the configuration at $t$ and the power functional (40), the correct set of equations at a subsequent time $t+\tau$ can be obtained by taking suitable variations at time $t+\tau$.

Proposition 5.1. The quasi-static equations in the interior of the elastic medium and of the fluid, as well as the correct balance of forces on the free boundary $\Gamma$, are obtained if the power functional (40) is stationary with respect to variations of $\hat{p}, \underline{v}$ in $\Sigma$ and $\underline{\underline{u}}_{h}$ in $\bar{\Omega}_{0}$ at time $t+\tau$, that satisfy

$$
\delta \underline{v}(t+\tau)=\frac{\delta \hat{u}_{h}(t+\tau)}{\tau} \circ \underline{\psi}^{-1}(t+\tau) \text { on } \Gamma
$$

REMARKS.

(1) This proposition is nothing but a reformulation of observation 2.1, where both $\delta E$ and the displacement $\underline{u}_{h}$ are multiplied by a factor $1 / \tau$.

(2) The addition "quasi-static" means that the deformations depend on time in such a way that the acceleration forces can be neglected.

(3) If variations of the domain $\Sigma$ are also considered, the Euler-Lagrange equations are not altered (in the present approximation) if the power density $W$ satisfies the inequality

$$
\tau W\left(\nabla_{x} \hat{\underline{\hat{v}}}\right) \underline{n} \ll\left(W^{\prime}\left(\nabla_{x} \hat{\underline{v}}\right)-\hat{p} I\right) \underline{n} \text { on } \Gamma .
$$

Indeed, the boundary term in the first variation of the power functional (40) is given by

$$
\int_{\Gamma} \delta \underline{v}(t+\tau) \cdot\left(W^{\prime}-\hat{p} I\right) \underline{n} \mathrm{~d} l-\int_{\Gamma_{0}} \frac{\delta \hat{u}_{h}}{\tau}(t+\tau) \cdot V^{\prime} \underline{n}_{0} \mathrm{~d} l_{0}+\int_{\Gamma} \frac{\delta \hat{u}_{h}}{\tau} \circ \underline{\psi}^{-1}(t+\tau) \underline{\tau} W \mathrm{~d} l .
$$


The last term can be dropped if (42) is satisfied. In general, it is not obvious that this condition is satisfied when $\tau$ tends to zero, since $\nabla \hat{v}$ depends on $\tau$. However, since $W$ is a quadratic function of $\nabla \hat{v}$ (approximation 3.1), one can conclude that this condition can always be fulfilled by taking $\tau$ small enough.

Furthermore, the power functional can be approximated in the same way as described in Section 4. That is

$$
\frac{E\left(\hat{u}_{h}(t+\tau)\right)-E\left(\hat{u}_{h}(t)\right)}{\tau}=\left.\frac{\gamma}{2 \tau} \int_{\Gamma} \hat{p} L(\hat{p}) \mathrm{d} x\right|_{t} ^{t+\tau}
$$

and at time $t+\tau$

$$
P(\hat{p}, \underline{\hat{v}})=\frac{1}{2} \int_{a}^{b}\left(\frac{\hat{h}^{3}}{12 \mu}\left(\partial_{x} \hat{p}\right)^{2}-\left(v_{k}+v_{0}\right) \hat{h} \partial_{x} \hat{p}-\mu \frac{\left(v_{k}-v_{0}\right)^{2}}{h}\right) \mathrm{d} x-\int_{\Gamma} \hat{p} \hat{v} \cdot \underline{n} \mathrm{~d} l .
$$

By introducing also the boundary condition (41), the following time-discretized description of the EHL problem is obtained.

PROPOSITION 5.2. In the approximation under consideration, the solution $\hat{p}(t+\tau), \hat{b}(t+\tau)$ of the EHL problem is a stationary point of the power functional

$$
\int_{a}^{\hat{b}}\left[\frac{h^{3}(\hat{p})}{12 \mu}\left(\partial_{x} \hat{p}\right)^{2}-\left(v_{k}+v_{0}\right) h(\hat{p}) \partial_{x} \hat{p}-\mu \frac{\left(v_{k}-v_{0}\right)^{2}}{h(p)}-(\hat{p}-\hat{p}(t)) \gamma L\left(\hat{\partial}_{t} \hat{p}\right)\right] \mathrm{d} x
$$

with

$$
h(\hat{p})=y_{0}-k_{0}-\frac{\beta r^{2}}{k_{0}}-\gamma L(\hat{p}), \quad \text { and } \quad \hat{\partial}_{t} \hat{p}=\left.\frac{\hat{p}(\alpha, t+\tau)-\hat{p}(\alpha, t)}{\tau}\right|^{\alpha=x(t+\tau)}
$$

for variations subject to $\hat{p}(a, \cdot)=\hat{p}(\hat{b}, \cdot)=0, \hat{p}(x, \cdot)>0$ for $a<x<b$ and $v_{k}=v_{0}$ at $x=\hat{b}$.

The Euler-Lagrange equation that results from variations of $\hat{p}(t+\tau)$ is

$$
\begin{aligned}
-\frac{1}{12 \mu} \partial_{x}\left(h^{3}(\hat{p}) \partial_{x} \hat{p}\right)+\partial_{x}\left(\frac{1}{2}\left(v_{k}+v_{0}\right)\right. & h(\hat{p}))-\gamma \hat{\partial}_{t} L(\hat{p}) \\
= & \frac{1}{2} L\left(\frac{1}{4 \mu} h^{2} \partial_{x} \hat{p}-\left(v_{k}+v_{0}\right) \partial_{x} \hat{p}+\mu\left(\left(v_{k}-v_{0}\right) / h\right)^{2}\right)
\end{aligned}
$$

The right-hand side results from variations of the fluid domain $\Sigma$. Those variations reduce in the present approximation to variations of the film thickness. As remarked before, this contribution to the Euler-Lagrange equation can be neglected. Indeed, the power functional is obtained by substituting the approximate extremizing velocity and displacement into the basic formulation given in proposition 5.1 .

A very illuminating observation is the following.

If the right-hand side is neglected, equation (44) is nothing but the law of conservation of mass of a volume $V=\left\{(\alpha, \beta) \in \mathbb{R}^{2} \mid x<\alpha<x+\Delta, 0<\beta<h(x, t)\right\}$, i.e.

$$
\frac{1}{\Delta} \frac{\mathrm{d}}{\mathrm{d} t} \iint_{V} \rho \mathrm{d} V=0 \text { for } \Delta \rightarrow 0
$$

Indeed, since the mass density $\rho$ is constant, it follows from (45) that

$$
0=\frac{1}{\Delta} \int_{\partial V} \underline{\hat{v}} \cdot n \mathrm{~d} l=\frac{1}{\Delta}\left(\left.\int_{0}^{\hat{h}(\cdot, t)} v_{1}(\cdot, y) \mathrm{d} t\right|_{x} ^{x+\Delta}+\int_{x}^{x+\Delta} \underline{\bar{v}} \cdot \underline{n}(x, k) \mathrm{d} x\right),
$$

The left-hand side of equation (44) is found by letting $\Delta \rightarrow 0$ in this expression. Concluding, it is found that proposition 5.2 can be interpreted as an (approximated) integrated form of the balance of mass.

One of the advantages of the time-discretized variational formulation is the fact that it leads to a numerical method in a natural way. Roughly speaking, the pressure is projected into a 
finite dimensional space, and the minimum of the power functional is calculated. Besides the formulation as an optimization problem, an important difference with existing numerical methods is that the iteration, starting from some initial value (for instance $p=0$ ), can be viewed as being time-like. A detailed description of the numerical method can be found in [13].

A final observation is that the first variation of the total power

$$
\mathscr{P}(p)=\int_{a}^{b}\left(\frac{h^{3}(p)}{12 \mu} p_{x}^{2}+\left(v_{k}-v_{0}\right) h(p) p_{x}-\mu \frac{\left(v_{k}-v_{0}\right)^{2}}{h(p)}\right) \mathrm{d} x
$$

vanishes if $p$ is the steady state solution (i.e. $\hat{\partial}_{t} p=0$ ) of equation (44). This proves the following proposition.

Proposition 5.3. The steady-state solution of the approximate EHL problem satisfies

$$
\operatorname{stat}_{p, b} \mathscr{P}(p),
$$

subject to the constraints of proposition 4.1 .

In this way a genuine, classical variational principle for the EHL problem is found. This provides another a posteriori justification for the proposed discrete-time principles.

\section{REFERENCES}

[1] A. Z. SZERI (Ed.), Tribology: Friction, Lubrication and Wear. Hemisphere, Washington (1980).

[2] L. MALVERN, Introduction to the Mechanics of a Continuous Medium. Prentice-Hall, New York (1969).

3] J. E. MARSDEN and J. R. HUGHES, Mathematical Foundations of Elasticity. Prentice-Hall, New York (1983).

[4] B. A. FINLAYSON, The Method of Weighted Residuals and Variational Principles: With Applications in Fluid Mechanics, Heat and Mass Transfer. Mathematics in Science and Engineering, Vol. 87. Academic Press, New York (1972).

[5] H. SCHLICHTING, Boundary Layer Theory (Translated from German), 6th edn. McGraw-Hill, New York (1968).

[6] J. C. COYNE and H. G. ELROD Jr, ASME J. Lubr. Technol. 92, 451 (1970).

[7] R. VERSTAPPEN, A variational formulation of a hydrodynamic lubrication problem. University of Twente, Memo No. 642 (1987).

(8) R. VERSTAPPEN and E. VAN GROESEN, ASME J. Tribol. 111, 108 (1989).

[9] G. CAPRIZ and G. CIMATTI, Free boundary problems in the theory of hydrodynamic lubrication. Free Boundary Problems: Theory and Applications (Edited by A. Fasano and M. Primicerio), Vol. 2, Research Notes in Mathematics 79, pp. 613-635. Pitman, Boston (1983).

[10] G. T. McALLISTER and S. M. ROHDE, JOTA 41, 599 (1983).

[11] A. E. H. LOVE, A Treatise on the Mathematical Theory of Elasticity, 4th edn, University Press, Cambridge (1952).

[12] R. GOHAR, Elastohydrodynamics. Ellis Horwood, Chichester (1988).

[13] $R$. VERSTAPPEN, The variational structure and numerical solution of an elastohydrodynamic lubrication problem. Submitted. 